\title{
Information assurance and advanced human-computer interfaces
}

Recent advances in wireless and ubiquitous technologies have led to a new dimension in mobile computing by extending the number of services and functionalities available. Wireless and Bluetooth technologies allow a wide spectrum of devices such as laptops, palmtops, PDA, intelligent sensors, and cell phones to communicate, process and exchange information. A new computing paradigm is growing up that allows users to access processed information anywhere and at any time.

Emerging wireless and ubiquitous networks typically involve interactions between a large number of entities (e.g. people, server, etc. ...) across different organizations. Information assurance comprises the techniques and solutions for information availability in wireless and ubiquitous networks. Information availability mechanisms compensate for failures in the network that result from either accidental breakdown or malicious attacks.

On the other hand, developments in software and hardware technologies are continuously driving application developers to growingly support our collaborative and communicative needs as social beings, both at work and at play. From these motivations it stems the idea behind this special issue, which focuses on intelligent interfaces that allow users to interact with mobile computers and systems seamlessly and naturally.

This special issue of Mobile Information Systems: An International Journal is devoted to recent developments in Information Assurance and Advanced Human-Computer Interfaces, such as those presented at the First International Workshop on Intelligent Interfaces for Human-Computer Interaction (IIHCI 2008), and at the 2008 Complex, Intelligent and Software Intensive Systems (CICIS-2008) International Conference, held on March 4-7 in Barcelona, Spain.

This issue includes 6 papers, classified into two broad groups: Information Assurance (2) and Advanced Human-Computer Interfaces (4). Brief synopses of the papers are given below.

Aikebaier, Enokido and Takizawa introduce the first of a group of two papers focusing on Information Assurance with wireless technologies, central in the development of ubiquitous systems. In particular, in "Design and evaluation of reliable data transmission protocol in wireless sensor network", they discuss a redundant data transmission protocol that improve fault-tolerance in sensor-actuator wireless networks. The second paper in this group is by Doci and Xhafa and is entitled: "WIT - a Wireless Integrated Traffic model". The authors address an important issue in ad-hoc wireless network pertaining the combined effect of traffic and mobility on overall network performance.

The second group of papers selected for this special issue addresses the role of interface for advanced human-computer interaction. In "EHeBby: an Evocative Humorist chatBot", Pilato, Augello, Vassallo and Gaglio discuss their approach to an evocative humorist chatting agent, capable of showing a specific sense of humor, enabling the development of chatting interfaces capable to amuse the users while performing their main content-providing task. Pirrone, Russo, Cannella and Peri, in "GAIML: a new 
language for verbal and graphical interaction in chatbots", look at a different conversational agent and language capable of dynamically adapting a graphical user interface depending on the specific application context. In his "Tangible interfaces for interactive multimedia presentations", Kanev presents a number of different ways to use physical objects to enhance multimedia presentations. As a last paper in this group, Durresi, Durresi, Merkoci, Barolli present a distributed system enabling global and ubiquitous health monitoring of patients. The interface integrates biomedical data with permanent medical information available at local Health medical Centers, enabling the appropriate actions to be planned and executed by medical staff.

As the Guest Editors of this issue, we would like to thank all the authors for their valuable contributions and the reviewers for their hard work under a tight schedule. The constructive comments of the reviewers were vital in selecting and improving the overall quality of the papers herein included.

Salvatore Vitabile and Antonio Gentile

University of Palermo, Italy

E-mail: salvatore.vitabile@gmail.com; gentile@unipa.it 

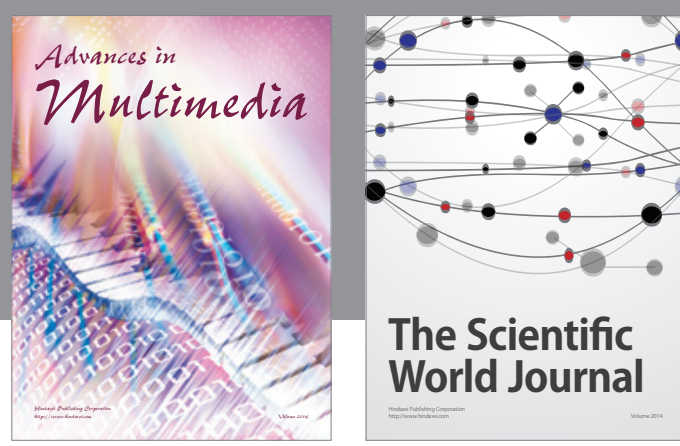

The Scientific World Journal
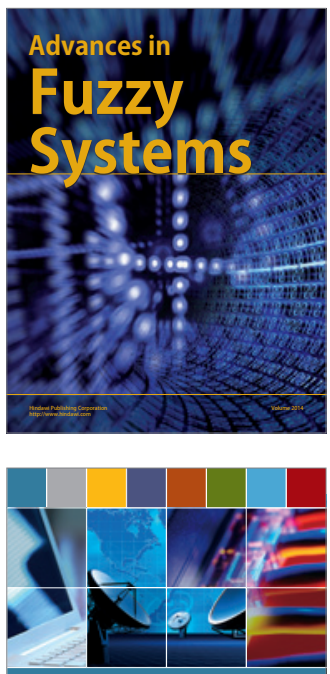

Computer Networks and Communications
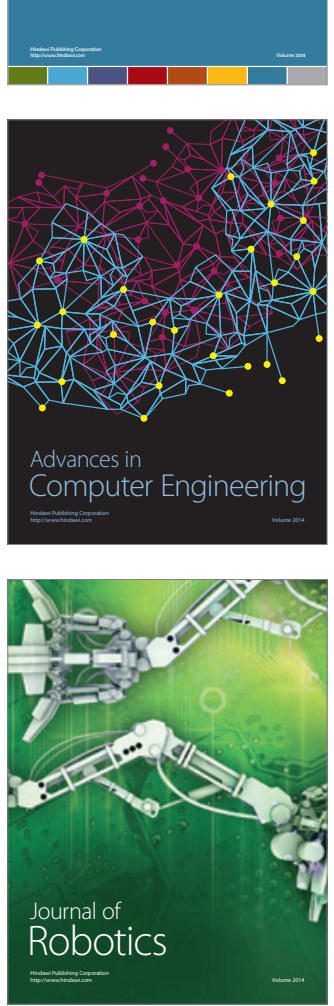
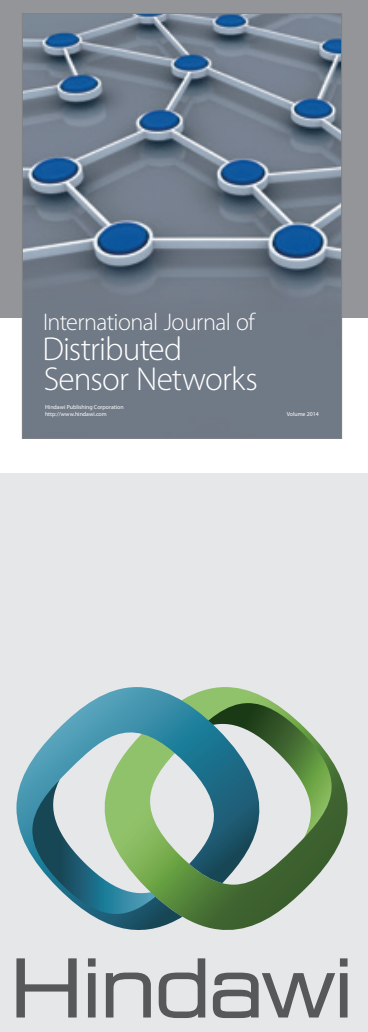

Submit your manuscripts at

http://www.hindawi.com
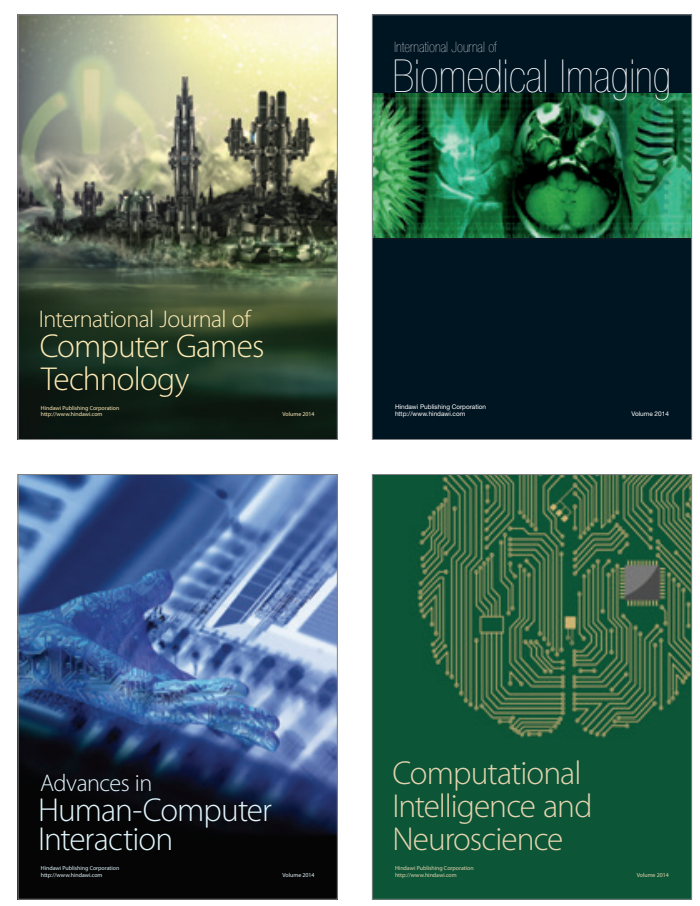
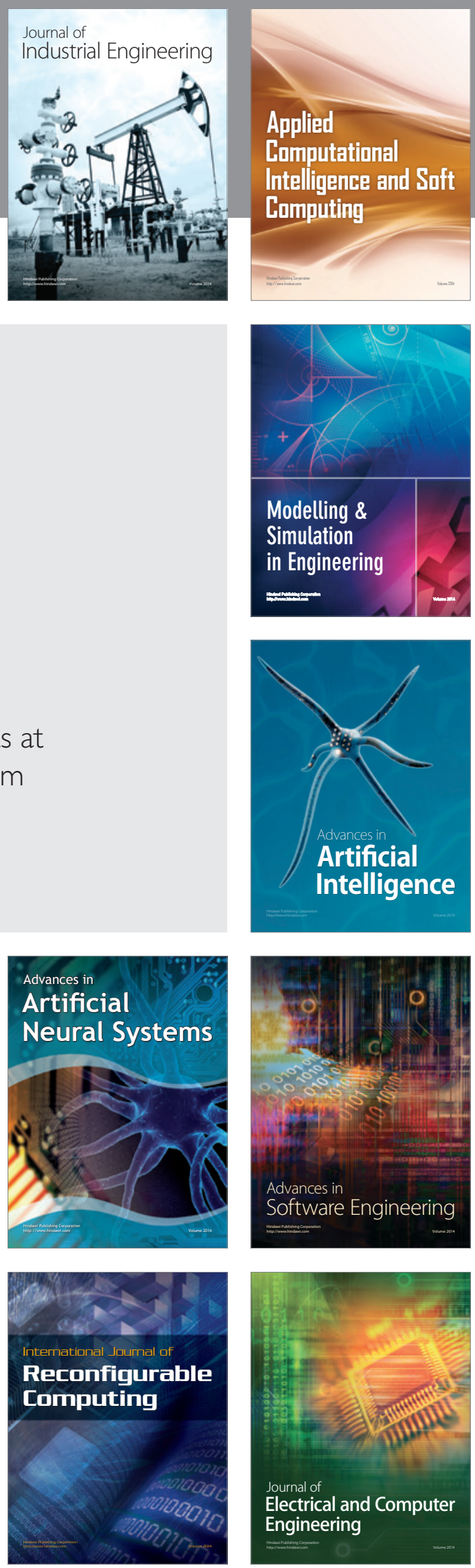